\title{
STUDY ON THE CURRENT STATE OF RESEARCH IN THE FIELD OF ARTIFICIAL CLAMPING SYSTEMS USING ASTRINGENT ELECTRO ADHESION TECHNOLOGY
}

\author{
Mihai-Nicolae Popescu ${ }^{1}$ and Mihai Avram² \\ 1,2 Politehnica University of Bucharest \\ mihai.p.nicolae@gmail.com, mavram02@yahoo.com
}

\begin{abstract}
This paper aims to provide an analysis of the current state of research in the field of artificial clamping systems using astringent electro adhesive technology.

The principle of electro adhesion phenomenon is described based on devices build as research prototypes made by several technology developers and research institutes.

Here is presented, starting with the first uses of electro adhesion devices in the 1960's as a standalone technology by NASA Research Center on astronaut garment for outer space, to the prehensile prototypes developed by Laboratory of Intelligent Systems, School of Engineering, Ecole Polytechnique Federale de Lausanne, used for gripping soft, fragile objects.

In this paper we show the various advantages of electro adhesion devices, also the limitations that depend on the proprieties of the adherent object.

Focusing on the reliability, the materials and the operating environment in the future commercial users(factories, logistics centers etc.) could envision the replacement of classic devices based on vacuum or magnetic technology with electro adhesive devices.
\end{abstract}

Keywords: Electro adhesion, Clamping System, Electro adhesive

\section{Introduction}

The electrification of bodies has been known since antiquity.

The Greek philosopher Thales of Miletus, who lived 2500 years ago (7th century BC), is the first to recall the phenomenon in his writings, where he presents experiments with pieces of amber which, when rubbed with a piece of fur, cause a electrical charge on its surface, attracting light objects [1].

The first commercial use of electro adhesion was at the paper-sheet fastening system of HP 9125A plotter (see Fig.1).

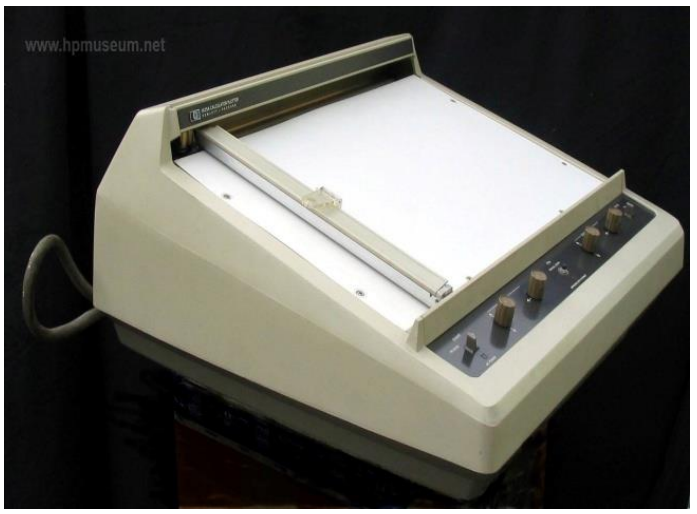

Figure 1: HP 9125A plotter
With the advancement of technology in the electronics industry, different uses of this phenomenon have appeared in the handling of fragile and scratch-sensitive objects.

In the field of artificial clamping systems with astringent action on objects, there are several types of technologies used:

- vacuum

- magnetic

- electro adhesive

"Electro adhesion is the electrostatic effect with astringent action between two surfaces subjected to an electric field" [2].

Electro adhesive phenomenon is expressed by the formula:

where:

$$
\frac{F}{A}=\frac{\epsilon V^{2}}{2 d^{2}}
$$

- $\mathrm{F}$ is the strenght between two charged electrodes separated by an insulator in the electro adhesive bond;

- $\epsilon$ is is the dielectric constant of the insulator;

- $\mathrm{d}$ is the measurement of electrode separation distance;

- A is the contact area "[3]

The principle of operation of electrically-adhesive devices varies according to the conductivity of the object over which prehension is exerted (properties 
represented by the simplified expression of Gauss's law):

a) for objects from dielectric materials $D=$ $(\epsilon E+P)$

b) for objects from conductive materials $D=\epsilon E$
The capability of supplying electric power to the electrodes by a circuit gives to the electro adhesion devices the advantage of electrically controllable adhesion technology by a single control voltage.

The constructive way encountered in experimental works is represented by conductive electrodes isolated in a polymer (Fig 2.).

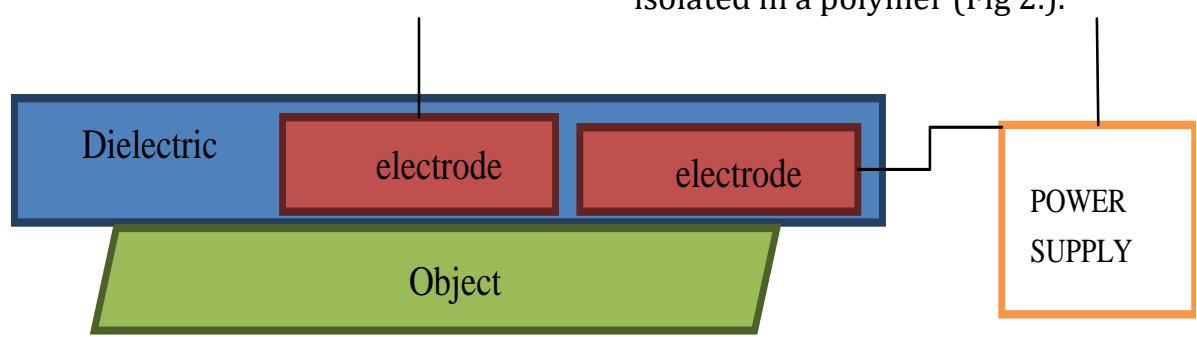

Figure 2: Graphical representation of electro adhesive pads

The electric fields set up opposite charges on the substrate when an electric charge is applied and thus the electrostatic adhesion between the electrodes and the induced loads on the substrate occurs.

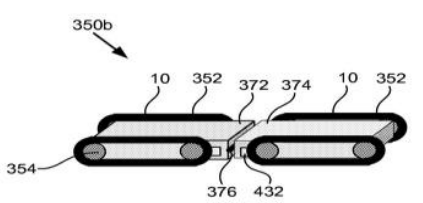

Described as a robot that uses for locomotion a mechanism that also comprises of an electro adhesive device, thus creating an adhesive force capable of obtaining vertical mobility.

\section{Applications of Electro Adhesive Phenomenon}

\subsection{Tracked / Wheeled Robots}

SRI international has developed and envisioned several types of wall climbing robots, one of them registered as patent US20100059298A1 (Fig.3).

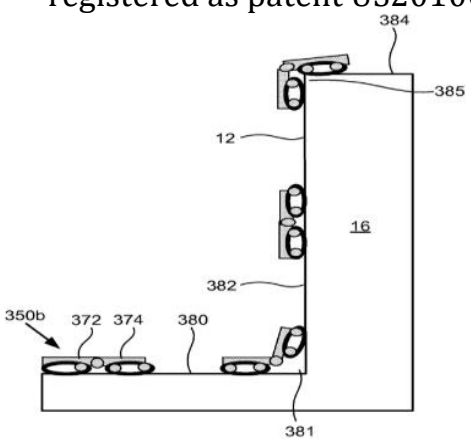

Figure 3: Graphical representation of the wall-crawling robot [5]

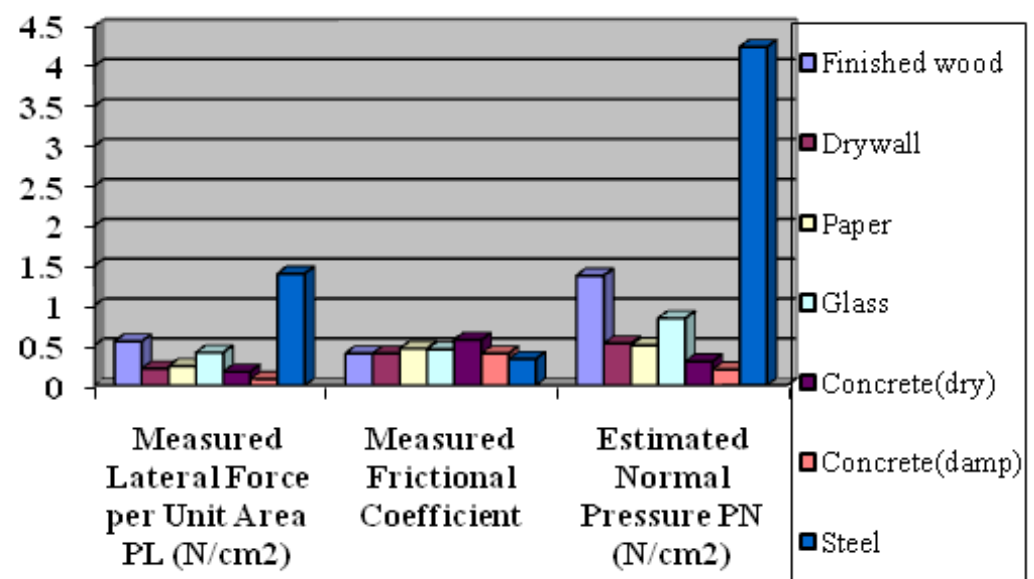

Figure 4: Clamping performance obtained on different materials [6] 


\subsection{Prehensile Devices}

\section{Garment application}

In the paper "Application Study of Electro adhesive Devices" by Richard P. Krape prepared by Chrisler Corporation Space Divison for National Aeronautics and Space Administration Langley Research Center [3], electro adhesion devices are started being researched and developed for their potential usefulness in prehension applications.

They used the electro adhesivity phenomenon in developing prototypes with multiple uses in aerospace garment applications as hand holds, knee pads, back and several other space suit accessories.

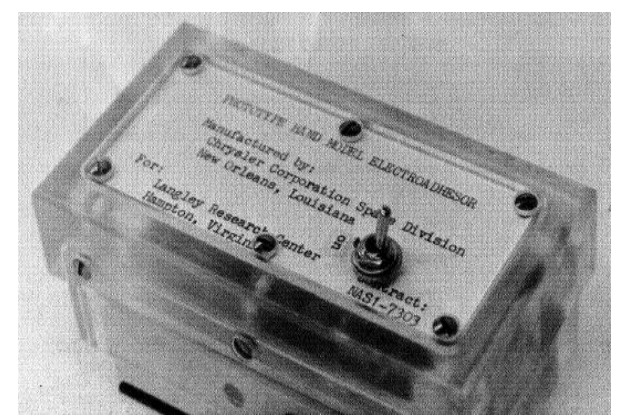

Figure 5: Hand-Model Electro adhesor Prototype Single Pole Type [3]

Specifications of the prototype (Fig. 5), at 1850 volts, the static pull force is $2,7 \mathrm{kgf}$ and the skid force is $18,14 \mathrm{kgf}$ with a battery longevity for operation of 42 hours.

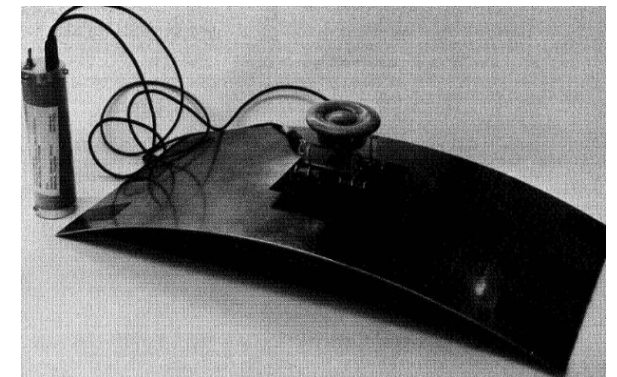

Figure 6: Flexible Prototype (Single-Pole) [3]

Specifications of the prototype (Fig. 6), at 1850 volts, the static pull force is $0,9 \mathrm{kgf}$ and the skid force is $0,4 \mathrm{kgf}$ with a battery longevity for operation of 40 hours.

Following their research, they determined that the resistivity, the chemical nature, the temperature, polarity of applied voltage, current density and the thickness of the electro adhesive coating influence the proprieties of electro adhesion devices directly.

\section{Stretchable electro adhesive pads}

Based on these proprieties electro adhesive devices have been developed in the form of stretchable electro adhesive pads. In this case, the forces that generate electro adhesion vary depending on how the pads are deformed [7].

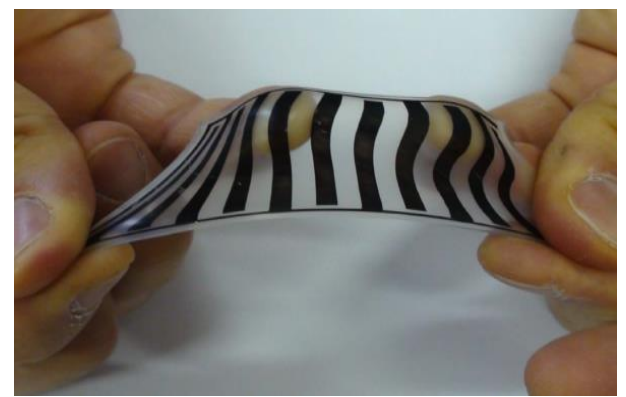

Figure 7: Stratchabl electro adhesive device[7]

If the electro adhesive pads are stretched, the space between electrodes increases, decreasing the electric field strength thus resulting in decreased polarization.

Stretchable electro adhesive devices are built in 2 stages using a rubber mixture having molding capability:

- first stage: the conductive rubber mixture containing Carbon black is poured into the mold obtaining the electrodes

- second stage: the electrodes are encapsulated using rubber poured over them as insulator.

\section{Soft grippers}

Using stretchable electro adhesive devices technology there has been developed a new method for creating soft grippers, enabled to operate deformable and fragile objects of any shape.

According to the creators of (Fig.8), the gripping force produced by electrostatic actuation is low (1 $\mathrm{mN})$, allowing operation of fragile objects, while the electro adhesion force can produce around $3.5 \mathrm{~N} / \mathrm{cm} 2$, thus enabling the capability of heavy objects lifting.

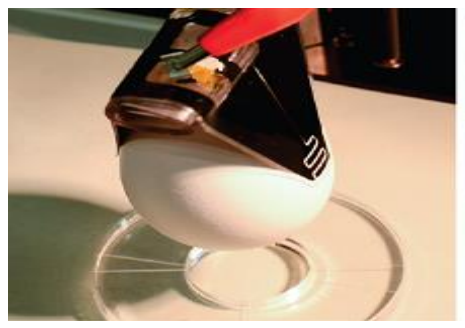

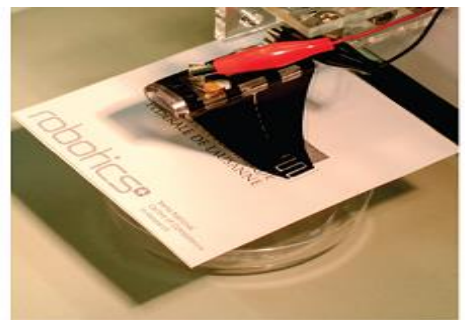

Figure 8: Soft grippers [8]

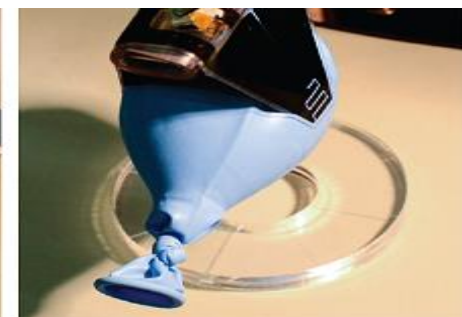




\section{Conclusion}

The purpose of this paper is to present the status of research in the domain of electroadhesion clamping devices, a short history on how this technology has been put to use, the advantages and the limitations of this technology as shown in Table 1.

Table 1. Comparison of electro adhesive technology with vacuum and magnetic

\begin{tabular}{|l|l|l|}
\hline Technology & Advantages & Limitations \\
\hline Vacuum & High adhesion forces on smooth surfaces & $\begin{array}{l}\text { Noisy, energy inefficient on rough } \\
\text { surfaces; requires separate pumping } \\
\text { source }\end{array}$ \\
\hline Magnetic & $\begin{array}{l}\text { Low-energy cost when not moving; quiet } \\
\text { operation; Proven high adhesion forces } \\
\text { on ferro magnetic materials. }\end{array}$ & $\begin{array}{l}\text { Technology available only for ferro } \\
\text { magnetic surfaces; high-energy cost } \\
\text { when moving; cannot climb some } \\
\text { plastic surfaces }\end{array}$ \\
\hline Electro adhesion & $\begin{array}{l}\text { Adheres to a large variety of surfaces; has } \\
\text { low-energy cost when mobile; it is quiet, } \\
\text { simple, lightweight, compliant; it can be } \\
\text { switched off for cleaning }\end{array}$ & $\begin{array}{l}\text { Requires very small amounts of } \\
\text { power ( 0.02 mW/N of weight } \\
\text { supported) to stay clamped }\end{array}$ \\
\hline
\end{tabular}

Worldwide it is estimated that in the future most jobs will be taken over by robots, but some of the jobs they will replace are hazardous jobs, by using them we will improve human wellbeing, this vision of future is very well described by Larry Page, Google general manager when he stated that "robotics will improve work and allow people to spend more time with their family"[9].

This is going to become a starting point for the authors in developing new robotic applications using the electro adhesive phenomenon.

\section{References}

[1] D.H. Trevena, Static fields in electricity and magnetism, Butterworths, London, (1961)

[2] Monkman G.J., Workpiece Retention during Machine Processing - Assembly Automation, Vol 20, issue 4, MCB University Press, (2000)

[3] Richard P. Krape, APPLICATIONS STUDY OF ELECTROADHESIVE DEVICES, Chrisler Corporation Space Divison for National Aeronautics and Space Administration Langley Research Center, (1968)

[4] Gauri Shivdas Gaunekar, IMPLEMENTATION OF ELECTROADHESION BASED ASTRICTIVE
PREHENSION SYSTEM, International Journal of Advanced Research in Electronics and Communication Engineering (IJARECE), Volume 5, Issue 11, (November 2016)

[5] Ronald E. Pelrine, Harsha Prahlad, Roy D. Kornbluh, Patrick D. Lincoln, Scott Stanford, SRI International. US Application, US20100059298A1

[6] H. Prahlad, R. Pelrine, S. Stanford, J. Marlow and R. Kornbluh, "Electro adhesive robots-wall climbing robots enabled by a novel, robust, and electrically controllable adhesion technology," 2008 IEEE International Conference on Robotics and Automation, Pasadena, CA, 2008, pp. 30283033.

[7] J. Germann, B. Schubert and D. Floreano, "Stretchable electro adhesion for soft robots," 2014 IEEE/RSJ International Conference on Intelligent Robots and Systems, Chicago, IL, 2014, pp. 3933-3938.

[8] J. Shintake, V. Cacucciolo, D. Floreano, H. Shea, Adv. Mater.2018, 1707035. https://doi.org/10.1002/adma.201707035

[9] https://www.theguardian.com/technology/2014 /jul/07/google-founders-larry-page-sergey-brininterview 